University of Nebraska - Lincoln

DigitalCommons@University of Nebraska - Lincoln

Timothy J. Gay Publications

Research Papers in Physics and Astronomy

March 2005

\title{
Angular momentum partitioning and the subshell multipole moments in impulsively excited argon ions
}

\author{
H. M. Al-Khateeb \\ University of Nebraska - Lincoln \\ B. G. Birdsey \\ University of Nebraska - Lincoln \\ Timothy J. Gay \\ University of Nebraska - Lincoln, tgay1@unl.edu
}

Follow this and additional works at: https://digitalcommons.unl.edu/physicsgay

Part of the Physics Commons

Al-Khateeb, H. M.; Birdsey, B. G.; and Gay, Timothy J. , "Angular momentum partitioning and the subshell multipole moments in impulsively excited argon ions" (2005). Timothy J. Gay Publications. 51.

https://digitalcommons.unl.edu/physicsgay/51

This Article is brought to you for free and open access by the Research Papers in Physics and Astronomy at DigitalCommons@University of Nebraska - Lincoln. It has been accepted for inclusion in Timothy J. Gay Publications by an authorized administrator of DigitalCommons@University of Nebraska - Lincoln. 


\title{
Angular momentum partitioning and the subshell multipole moments in impulsively excited argon ions
}

\author{
H. M. Al-Khateeb, ${ }^{1,2}$ B. G. Birdsey, ${ }^{1, *}$ and T. J. Gay ${ }^{1}$ \\ ${ }^{1}$ Behlen Laboratory of Physics, University of Nebraska, Lincoln, Nebraska 68588-0111, USA \\ ${ }^{2}$ Department of Physics, Jordan University of Science \& Technology, Irbid, P.O. Box 3030, 22110 Jordan \\ (Received 27 August 2003; revised manuscript received 7 September 2004; published 10 March 2005)
}

\begin{abstract}
We have investigated collisions between transversely polarized electrons and $\mathrm{Ar}$, in which the $\mathrm{Ar}$ is simultaneously ionized and excited to the $\operatorname{Ar}^{+*}\left[3 p^{4}\left({ }^{1} D\right) 4 p\right]$ states. The Stokes parameters of the fluorescence emitted in the following transitions was measured: $\left({ }^{1} D\right) 4 s^{2} D_{5 / 2}-\left({ }^{1} D\right) 4 p^{2} F_{7 / 2}(461.0 \mathrm{~nm}),\left({ }^{1} D\right) 4 s^{2} D_{5 / 2}$ $-\left({ }^{1} D\right) 4 p^{2} F_{5 / 2} \quad(463.7 \mathrm{~nm}), \quad\left({ }^{1} P\right) 3 d^{2} D_{5 / 2}-\left({ }^{1} D\right) 4 p^{2} D_{5 / 2} \quad(448.2 \mathrm{~nm}), \quad$ and $\quad\left({ }^{1} D\right) 4 s^{2} D_{3 / 2}-\left({ }^{1} D\right) 4 p^{2} P_{3 / 2}$ $(423.7 \mathrm{~nm})$. We develop the angular momentum algebra necessary to extract from these data, starting from the overall atomic $J$ multipoles, the partitioning of orbital angular momentum into the ${ }^{1} D$ core electric quadrupole and hexadecapole moments, and the outer $4 p$ electric quadrupole moment. The magnetic dipole of the outer electron is also determined. This procedure requires the assumption of good $L S$ coupling for these states, which is justified. We recouple these individual core- and outer-electron moments to calculate the initial electric quadrupoles, hexadecapoles, and hexacontatetrapoles of the initial excited-state manifold. The detailed time structure of the electron-atom collision is considered, as well as the time evolution of the excited ionic state. The Rubin-Bederson hypothesis is thus shown to hold for the initial ionic $L$ and $S$ terms. The consequences of the breakdown of $L S$ coupling are considered. From the circular polarization data, estimates of the relative importance of direct and exchange excitation cross section are made. We discuss experimental issues related to background contributions, Hanle depolarization of the fluorescence signal, and cascade contributions. Nonlinearity of the equations relating the Stokes parameters to the subshell multipole moments complicates the data analysis. Details of the Monte Carlo terrain-search algorithm used to extract multipole data is discussed, and the implications of correlation between the various subshell multipole moments is analyzed. The physical significance of the higher-order multipole moments is discussed, and graphical representations of the effects of these multipoles on the excited ionic charge clouds is presented.
\end{abstract}

DOI: 10.1103/PhysRevA.71.032707

PACS number(s): 34.80.Dp, 34.80.Nz, 34.80.Pa

\section{INTRODUCTION}

Studies of electron scattering by atoms have most often involved the measurement of cross sections for the various collision channels: elastic scattering, target excitation, and ionization with or without excitation of the target [1]. As experimental techniques have become increasingly sophisticated, more esoteric quantities such as target orientation and alignment, complex scattering amplitudes, and spin polarization of the scattered electron and/or target atom have been measured [2]. Crafty theorists and the advent of high-speed computers have pushed the state-of-the-art for calculation of scattering parameters to an impressive level. We are now at the point where the most basic many-body long-range-force problem, electron-hydrogen scattering, is solved $[3,4]$. Our understanding of scattering by the light alkalis and helium is also in very good shape.

One of the remaining problems in electron-atom scattering is dealing with targets having many equivalent electrons in the ground state, e.g., the heavy noble gases (HNG's). While such targets have been studied extensively in the past, it has only been recently that collision theory had a hope of dealing with such measurements comprehensively [5]. The

*Present address: School of Physics, University of Western Australia, Perth, Western Australia 2009, Australia. complexity of these targets, while a bane to theorists, offers a richer variety of collision physics to be studied.

The subject of the work reported here involves a qualitatively new level of detail in terms of investigating the residual target in an electron-atom collision. We have made the first measurements of the electromagnetic multipole moments of individual target subshells as opposed to its overall moments. (A preliminary report on this work appeared several years ago [6].) Such a study is possible because of the structural complexity of the target's final state and, simultaneously, its simple angular momentum coupling scheme. We have studied the reaction

$$
\begin{aligned}
e^{-}(\uparrow)+\operatorname{Ar}\left[3 p^{6}\left({ }^{1} S_{0}\right)\right] & \rightarrow \operatorname{Ar}^{* *}\left[3 p^{4}\left({ }^{1} D\right) 4 p\right]+2 e^{-} \\
& \rightarrow \operatorname{Ar}^{* *}\left[3 p^{4}\left({ }^{1} D\right) 4 s \text { or } 3 p^{4}\left({ }^{1} D\right) 3 d\right]+\gamma
\end{aligned}
$$

using transversely polarized electrons. The scattered electrons were not detected; the polarization of the fluorescence emitted by the residual ion was determined for those photons emitted along the electron polarization axis. Such experiments are often referred to as "integrated Stokes parameter measurements." In the late 1960s and 1970s, excitation or ionization collisions of this type were studied extensively because of their relevance to the operation of Ar ion lasers (see, e.g., Refs. [7-11]). More recently, they have come un- 
der renewed scrutiny because of the enhanced role played by correlation between the outgoing electrons in this channel $[12,13]$. In addition, detection of the fluorescence emitted by the residual excited states provides higher experimental energy resolution than that available from direct $(e, 2 e)$ measurements.

The goal of these experiments is to determine the electric and magnetic multipole moments of the $4 p$ excited electron, and, separately, the electric multipole moments of the ${ }^{1} D$ core, as prepared during the collision. The extraction of these moments relies on the important assumption that the collision time, during which the spin and orbital angular momenta of the core and outer electron become well defined, is short compared with the time required for the core and $4 p$ electron to dynamically couple to form the total $L, S$, and $J$ of the residual ion. We refer to this assumption as the RubinBederson (RB) hypothesis, because they were the first to state it generically and explicitly [14]. (The same physical assumptions are often referred to mistakenly as the "Percival-Seaton" hypothesis for historical reasons that remain obscure $[15,16]$.) Using the RB hypothesis, one can infer the values of the subshell multipole moments by determining a sufficient number of multipole moments associated with various $J$ states of the coupled system. The ancillary assumption of $L S$ coupling of the residual ionic state is not necessary for our analysis, but does make it much simpler.

The ionization or excitation channel for Ar targets is a good proof-of-principle system for this type of analysis for several reasons. The residual Ar II states are well $L S$ coupled, well separated in energy, and fluoresce at wavelengths predominantly above $400 \mathrm{~nm}$. This allows for narrowbandwidth interference filters to be used to separate the various transitions from the same manifold of a given configuration. The ionic core, having two $3 p$ holes, can couple to form three angular momentum configurations. These provide a variety of possible measurements. The threshold for the ionic states in question is large $(>30 \mathrm{eV})$, so control of the incident electron beam is straightforward. Finally, Ar is an easy target with which to work experimentally.

\section{THE STRUCTURE OF Ar II AND THE RUBIN-BEDERSON HYPOTHESIS}

Let us consider the Grotrian diagram for the $\operatorname{Ar} \mathrm{II}\left(3 p^{4} 4 p\right)$ configuration, shown in Fig. 1. The excited states of this configuration have energies varying between 19.2 and $23.9 \mathrm{eV}$ above the $3 p^{5}{ }^{2} P_{3 / 2}$ ground state of $\mathrm{Ar}$ II, or 35.0 to $39.6 \mathrm{eV}$ above the neutral ground state. These states are generally quite well $L S$ coupled [11]. In the case of states with a (predominantly) ${ }^{1} D$ core, the ${ }^{2} P_{1 / 2}$ and ${ }^{2} P_{3 / 2}$ states have $\sim 15 \%{ }^{3} P$ core character (see Table I). Considering this structure, we can assign rough values of "relaxation" or "coupling" time to the various angular momenta of the ionic constituents. These angular momenta are $l_{3 p}$ and $l_{3 p}^{\prime}$, the individual core-hole orbital angular momenta, their corresponding spins $s_{3 p}$ and $s^{\prime}{ }_{3 p}$, respectively, and the same quantities associated with the outer $4 p$ electron, $l_{o}$ and $s_{o}$. The energy splittings of the various configuration manifolds are

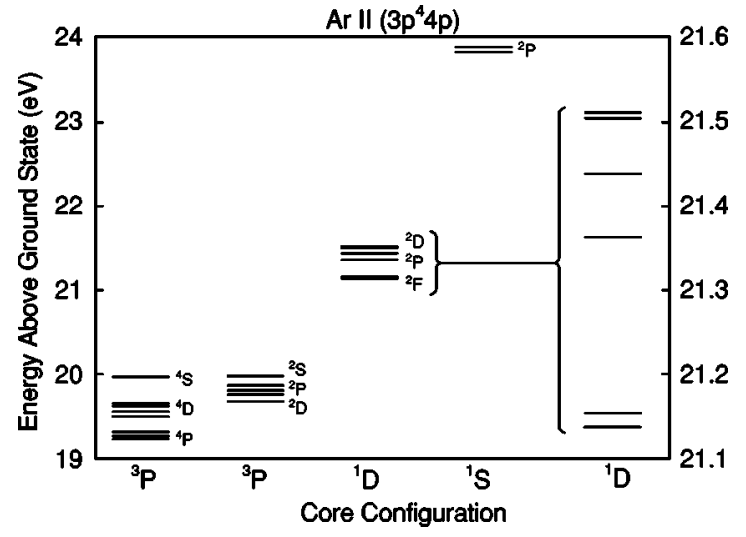

FIG. 1. The $\operatorname{Ar}$ II $\left(3 p^{4} 4 p\right)$ states. Those considered in this work are shown on an expanded scale at the right. Energies indicated are relative to the $3 p^{5}{ }^{2} P_{3 / 2}$ Ar II ground state.

connected to these times by the energy-time form of the Heisenberg uncertainty relation relevant for angular momentum coupling and its attendant fine structure [17]:

$$
\Delta E \Delta t=h .
$$

The structure of Fig. 1 indicates that the strongest coupling in the residual ion is that of the Coulombic interaction between the two core holes; $l_{3 p}$ and $l^{\prime}{ }_{3 p}$ couple to form $l_{c}$ over a time corresponding to the energy range of core states within the configuration: $\sim 4 \mathrm{eV}$ or $\sim 10^{-15} \mathrm{~s}$. The coupling model we are using in this discussion cannot distinguish between core $l$ coupling and core $s$ coupling. Thus one could argue that the core spins couple to form $s_{c}$ within this time as well. This is somewhat redundant, however, given that $l_{c}$, comprising two equivalent holes, determines $s_{c}$. Having decided upon a core coupling, $l_{c}$ now couples with $l_{o}$ to form $L$ in a time corresponding to the typical splitting of a given core manifold: $\sim 0.5 \mathrm{eV}$, or $\sim 8 \times 10^{-15} \mathrm{~s}$. The $s_{c}-s_{o}$ coupling time can be estimated by considering the splittings between total spin $S=1 / 2$ and $S=3 / 2$ levels having the same core and value of $L$. These splittings are $\sim 0.2 \mathrm{eV}$ between the $\left({ }^{3} P\right)^{4} D$ and $\left({ }^{3} P\right)^{2} D$ states ranging up to $\sim 0.5 \mathrm{eV}$ between the $\left({ }^{3} P\right)^{4} P$ and $\left({ }^{3} P\right)^{2} P$ levels. Thus the coupling times, depending on $L$, vary between $\sim 8 \times 10^{-15}$ s and $2 \times 10^{-14}$ s. Finally, $L$ and $S$ couple in a time corresponding to the fine-structure multiplet splitting. For the ${ }^{1} D$ core system, which is our focus in this paper, this ranges between $6 \mathrm{meV}$ and $75 \mathrm{meV}$ for the ${ }^{2} D$ and ${ }^{2} P$ multiplets, respectively. The final $J$ state of the ion in the ${ }^{1} D$ core system is thus established in a time ranging between $\sim 6 \times 10^{-14} \mathrm{~s}$ and $7 \times 10^{-13} \mathrm{~s}$, respectively. The above discussion is represented schematically in the diagram shown in Fig. 2.

There are two other relevant time scales in the problem: the decay time of these excited states, and the time of the collision which produces them. The lifetimes of the $4 p$ states against decay to the $3 d$ and $4 s$ levels are greater than $3 \mathrm{~ns}$ in all cases [18]. Thus the fluorescence we observe is from completely relaxed states. Collision times can be estimated as follows. We consider incident electrons of energy $39 \mathrm{eV}$, roughly $1 \mathrm{eV}$ above the average excitation threshold of the 
TABLE I. Parentage of the $\operatorname{Ar} I\left(3 p^{4} 4 p\right)$ states with a predominantly ${ }^{1} D$ core. Data of Ref. [11].

\begin{tabular}{|c|c|c|c|c|c|c|c|c|c|}
\hline \multirow[b]{2}{*}{ State label } & \multicolumn{9}{|c|}{ Pure Russell-Saunders states } \\
\hline & $\left({ }^{3} P\right)^{4} D$ & $\left({ }^{3} P\right)^{4} P$ & $\left({ }^{3} P\right)^{4} S$ & $\left({ }^{3} P\right)^{2} D$ & $\left({ }^{3} P\right)^{2} P$ & $\left({ }^{3} P\right)^{2} S$ & $\left({ }^{1} D\right)^{2} P$ & $\left({ }^{1} D\right)^{2} D$ & $\left({ }^{1} D\right)^{2} F$ \\
\hline$\left({ }^{1} D\right)^{2} F_{7 / 2}$ & 0.0030 & & & & & & & & 0.9970 \\
\hline$\left({ }^{1} D\right)^{2} F_{5 / 2}$ & 0.0007 & & & 0.0026 & & & & 0.0035 & 0.9932 \\
\hline$\left({ }^{1} D\right)^{2} D_{5 / 2}$ & 0.0004 & 0.0011 & & 0.0024 & & & & 0.9928 & 0.0033 \\
\hline$\left({ }^{1} D\right)^{2} D_{3 / 2}$ & 0.0003 & 0.0002 & & 0.0004 & 0.0002 & & 0.0001 & 0.9970 & \\
\hline$\left({ }^{1} D\right)^{2} P_{3 / 2}^{3 / 2}$ & & 0.0002 & 0.0025 & 0.0002 & 0.1196 & & 0.8774 & 0.0001 & \\
\hline$\left({ }^{1} D\right)^{2} P_{1 / 2}$ & & 0.0001 & & & 0.1501 & 0.0009 & 0.8490 & & \\
\hline
\end{tabular}

states we are considering. The ionic radius of Ar II is 2.9 a.u. When the incident electron is 5 radii away from the target's nucleus, the electric field due to it at the target is a factor of 25 less than the corresponding field when the incident electron is at one ionic radius. The time required for a $39 \mathrm{eV}$ electron to travel from five radii out and traverse the target diameter is 15 a.u., or about $3.6 \times 10^{-16} \mathrm{~s}$. We now assume that the two free electrons exit the space of the residual ion in such a way that they share the excess energy equally, with both having asymptotic energies of $0.5 \mathrm{eV}$. Taking the residual ionic charge felt by one electron to be $1 / 2 e$ (to account roughly for the effect of shielding of one outgoing electron by the other), the time required for the electrons to move to a distance of 5 ionic radii from one ionic radius is another 30 a.u. Thus the total collision time over which the electric field at the target increases from $1 / 25$ of its maximum value and then returns to this value is $\sim(1-2) \times 10^{-15} \mathrm{~s}$.

This rather conservative estimate implies that the residual ionic core is formed over a time comparable to the collision time itself, so that $l_{3 p}$ and $l_{3 p}^{\prime}$ are never really well-defined vector quantities. However, the collision is essentially complete well before the core and $4 p$ angular momenta relax, implying that these quantities should be well defined, and will couple separately to produce the complete ${ }^{1} D$ core manifold (Fig. 2). To put this another way, the Rubin-Bederson

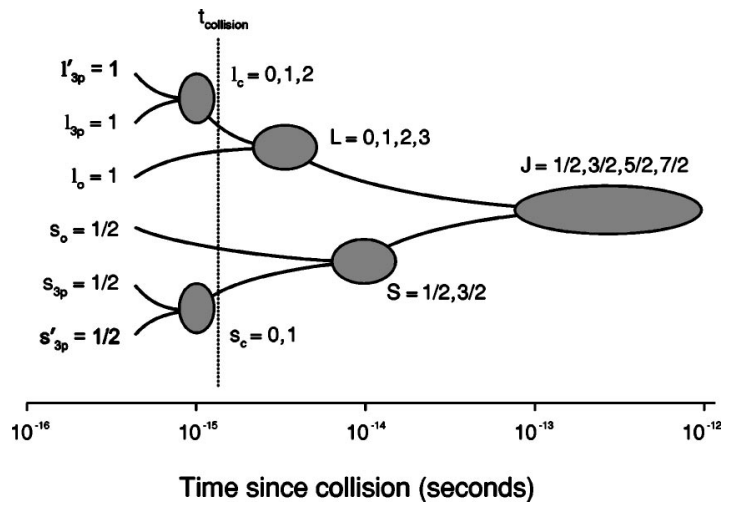

FIG. 2. The angular momentum coupling times of the $\operatorname{Ar}$ II $\left(3 p^{4} 4 p\right)$ states (see text). Vertical dotted line indicates the collision time. The horizontal width of the compound states, indicated by the ovals, represents the range of coupling times for the whole configuration. hypothesis says that, in this system, all the ${ }^{1} D$ core terms can be characterized by the same multipoles of $l_{c}, l_{o}$, and $s_{o}$.

\section{ANGULAR MOMENTUM ALGEBRA}

The goal of these experiments is to completely characterize the excited states produced in the collisions we observe. Because we do not detect the scattered electrons, we cannot determine pure quantum-mechanical complex amplitudes, but must settle instead for the reduced tensor multipole moments of the excited-state density matrix. As we shall see, this loss of information in one regard will allow us to obtain more complete information about the individual subshells of the residual ion. In the $L S$-coupling approximation, applied both to the collision complex as a whole and the relaxed residual ion, we can determine all of the allowable electric and magnetic multipole moments of both the $4 p$ outer shell and the ${ }^{1} D$ core. We take the incident beam to be along $\hat{z}$ and the electron polarization to be along $\hat{y}$ of a right-handed coordinate system. Given the axial symmetry of the collision geometry and excitation by transversely polarized electrons, the nonzero moments are all relevant monopoles, the electric quadrupole and hexadecapole of the core along $\hat{z}$, the electric quadrupole of the $4 p$ electron along $\hat{z}$, and the magnetic dipole of the latter along the electron polarization axis. If $L S$ coupling fails, the quadrupole and hexadecapole moments can have components along $(\hat{x}+\hat{z})$ as well $[19,20]$. Given the integrated nature of our experiment, knowledge of these multipoles characterizes to the fullest possible extent the excited states of the residual ion.

We now connect the observables in these experiments, the three normalized integrated Stokes parameters of the fluorescence, with the multipoles enumerated above. The expressions for the linear polarization along $\hat{z}, P_{1}$, the linear polarization along $\hat{x}+\hat{z}, P_{2}$, and $P_{3}$, the circular polarization are [21]

$$
\begin{gathered}
P_{1} \equiv \frac{I\left(0^{\circ}\right)-I\left(90^{\circ}\right)}{I\left(0^{\circ}\right)+I\left(90^{\circ}\right)}=\sqrt{\frac{3}{2}}\left\{\begin{array}{lll}
1 & 1 & 2 \\
J & J & J_{f}
\end{array}\right\}\left\langle T(J)_{20}\right\rangle / I, \\
P_{2} \equiv \frac{I\left(45^{\circ}\right)-I\left(135^{\circ}\right)}{I\left(45^{\circ}\right)+I\left(135^{\circ}\right)}=\sqrt{\frac{3}{2}}\left\{\begin{array}{lll}
1 & 1 & 2 \\
J & J & J_{f}
\end{array}\right\} \operatorname{Re}\left\langle T(J)_{21}\right\rangle / I,
\end{gathered}
$$




$$
P_{3} \equiv \frac{I^{+}-I^{-}}{I^{+}+I^{-}}=-\sqrt{\frac{3}{2}}\left\{\begin{array}{ccc}
1 & 1 & 1 \\
J & J & J_{f}
\end{array}\right\} \operatorname{Im}\left\langle T(J)_{11}\right\rangle / I,
$$

with

$I \equiv\left(I^{+}+I^{-}\right)=\frac{2(-1)^{J+J_{f}}}{3 \sqrt{2 J+1}}\left\langle T(J)_{00}\right\rangle+\sqrt{\frac{1}{6}}\left\{\begin{array}{ccc}1 & 1 & 2 \\ J & J & J_{f}\end{array}\right\}\left\langle T(J)_{20}\right\rangle$,

where $I(\theta)$ is the intensity of light linearly polarized along the axis making an angle $\theta$ with $\hat{z}$ in the $x-z$ plane, $I^{+(-)}$is the intensity of light with positive (negative) helicity, the $\{\ldots\}$ are $6 j$ coefficients, $J$ is the total angular momentum of the excited state, and $J_{f}$ corresponds to the state to which it decays. The $\left\langle T(J)_{K Q}\right\rangle$ 's are the irreducible tensor components of the excited-state density matrix written in the $\left|J M_{J}\right\rangle$ basis, with the brackets indicating that the scattered electrons are not observed. Thus $\left\langle T(J)_{00}\right\rangle$ is proportional to the total cross section for exciting the state in question, $\operatorname{Im}\left\langle T(J)_{11}\right\rangle$ is proportional to its magnetic dipole moment (or "orientation") along the $\hat{y}$ axis, and $\left\langle T(J)_{20}\right\rangle$ and $\left\langle T(J)_{21}\right\rangle$ are related to the electric quadrupole moments (or "alignments") along $\hat{z}$ and $\hat{x}+\hat{z}$, respectively. Note that the measurement of photons from a given transition can only yield information about atomic $J, M_{J}$ moments with rank 2 and lower.

Under the assumption of $L S$ coupling for the ion and the validity of the Rubin-Bederson hypothesis, i.e., that the collision time is much shorter than the $L S$ relaxation time, we can write

$$
\left\langle T(J)_{K Q}\right\rangle=\sum_{k q, k^{\prime} q^{\prime}} \beta_{K Q, k q, k^{\prime} q^{\prime}}^{J, L, S}\left\langle T(L)_{k q}\right\rangle\left\langle T(S)_{k^{\prime} q^{\prime}}\right\rangle
$$

The constants $\beta_{\ldots}^{\ldots}$ are recoupling coefficients, defined as

TABLE II. Coefficients relating the Stokes parameters $P_{1}$ and $P_{3}$ to the electric quadrupole and magnetic dipole moments in Eqs. (11) and (12). LS coupling is assumed.

\begin{tabular}{lcrcrrrr}
\hline \hline State & $a^{1}$ & \multicolumn{1}{c}{$b^{1}$} & $c^{1}$ & \multicolumn{1}{c}{$a^{3}$} & $b^{3}$ & \multicolumn{1}{c}{$c^{3}$} & $d^{3}$ \\
\hline${ }^{2} F_{7 / 2}$ & 3 & -6.4657 & 1 & -5.8773 & 1.6979 & -6.4657 & 1 \\
${ }^{2} F_{5 / 2}$ & 3 & 5.0520 & 1 & -4.5922 & 3.2265 & 5.0520 & 1 \\
${ }^{2} D_{5 / 2}$ & 3 & 5.2293 & 1 & 1.4794 & -0.3536 & 5.2293 & 1 \\
${ }^{2} D_{3 / 2}$ a & 3 & -23.9603 & 1 & 10.1426 & -8.4859 & -23.9603 & 1 \\
${ }^{2} P_{3 / 2}$ & 3 & 5.9759 & 1 & 2.8170 & -0.4715 & 5.9759 & 1 \\
${ }^{2} P_{1 / 2}{ }^{b}$ & & & & -0.2357 & 0.0000 & 1.0000 & 0 \\
\hline \hline
\end{tabular}

${ }^{\mathrm{a}}$ The coefficients for this state are related to the transition which has a wavelength of $449.1 \mathrm{~nm}$ [i.e., it has $3 p^{4}\left({ }^{3} P\right) 3 d^{2} D_{5 / 2}$ as a final state].

${ }^{\mathrm{b}}$ The coefficients for this state are related to the transition which has a wavelength of $413.2 \mathrm{~nm}$ [i.e., it has $3 p^{4}\left({ }^{3} D\right) 4 s^{2} D_{3 / 2}$ as a final state].

$$
\begin{aligned}
\beta_{K Q, k q, k^{\prime} q^{\prime}}^{J, L, S}= & (2 J+1)\left[(2 k+1)\left(2 k^{\prime}+1\right)\right]^{1 / 2} \\
& \times\left\{\begin{array}{ccc}
L & S & J \\
L & S & J \\
k & k^{\prime} & K
\end{array}\right\}\left(k q, k^{\prime} q^{\prime} \mid K Q\right),
\end{aligned}
$$

where $(\mid)$ is a Clebsch-Gordan coefficient and \{\} is a $9 j$ coefficient. The two assumptions mentioned above are embodied mathematically in the way we write the product of the $L$ and $S$ multipoles in Eq. (7). If these multipoles were correlated, either by virtue of strong magnetic forces in the residual ion leading to the breakdown of $L S$ coupling, or by the fact that they were produced in a correlated manner during the collision, we would have to write them as a correlated product: $\langle T(L) \otimes T(S)\rangle$.

The experimental geometry and the assumption of $L S$ coupling during the collision allow only $\left\langle T(S)_{00}\right\rangle,\left\langle T(S)_{11}\right\rangle$, and $\left\langle T(L)_{K 0}\right\rangle$ (with $K$ even) to be nonzero. Equation (7) thus has the general form

$$
\begin{gathered}
\left\langle T(J)_{00}\right\rangle=a\left\langle T(L)_{00}\right\rangle\left\langle T(S)_{00}\right\rangle, \\
\left\langle T(J)_{11}\right\rangle=b\left\langle T(L)_{00}\right\rangle\left\langle T(S)_{11}\right\rangle+c\left\langle T(L)_{20}\right\rangle\left\langle T(S)_{11}\right\rangle, \\
\left\langle T(J)_{20}\right\rangle=d\left\langle T(L)_{20}\right\rangle\left\langle T(S)_{00}\right\rangle,
\end{gathered}
$$

and

$$
\left\langle T(J)_{21}\right\rangle=0,
$$

where $a, b, c$, and $d$ are real constants. We note that $P_{1}$ is solely a function of $\left\langle T(J)_{20}\right\rangle /\left\langle T(J)_{00}\right\rangle$. This ratio, given Eq. (9), is equal to $\left\langle T(L)_{20}\right\rangle /\left\langle T(L)_{00}\right\rangle$. Moreover, $P_{3}$ depends only on this ratio and $\left\langle T(S)_{11}\right\rangle /\left\langle T(S)_{00}\right\rangle$. We can simplify our notation significantly by defining "normalized multipoles"

$$
\left\langle T(X=Y)_{k q}\right\rangle /\left\langle T(X=Y)_{00}\right\rangle \equiv X(Y)_{k q} .
$$

Here $X$ is the angular momentum quantity in question and $Y$ is its numerical value which may or may not be specified. (Generally we will specify $Y$ for orbital angular momenta $L$, where it can take on different values, but suppress it for $S, l_{\mathrm{o}}$, $l_{c}$, and $s_{o}$ which have unique values in this work.) The ionic electric multipoles are further limited to those having $K$ $\leqslant 2 L$.

We can thus write

$$
P_{1}(J)=\frac{a_{J L}^{1} L(L)_{20}}{b_{J L}^{1}+c_{J L}^{1} L(L)_{20}}
$$

and

$$
P_{3}(J)=\frac{a_{J L}^{3}+b_{J L}^{3} L(L)_{20}}{c_{J L}^{3}+d_{J L}^{3} L(L)_{20}} S_{11}
$$

where the coefficients are given in Table II for the various possible combinations of $L$ and $J$. It is apparent from the structure of Eqs. (11) and (12) that for the purposes of deducing the $L(L)_{20}$ and $S_{11}$, measurement of $P_{1}$ and $P_{3}$ for a single $L$-state fine-structure multiplet term is sufficient. Measurement of other (algebraically) redundant terms serves, however, as a check of the RB hypothesis. We limit our 
consideration to the ${ }^{2} F_{7 / 2},{ }^{2} F_{5 / 2},{ }^{2} D_{5 / 2}$, and ${ }^{2} P_{3 / 2}$ states, which will prove sufficient for the determination of all nonzero multipoles. The choice of these specific terms depends on experimental details, to be discussed in Sec. V.

We note that Eq. (7), when evaluated for $\left\langle T(J)_{21}\right\rangle$, gives an expression that is identically zero. This is a consequence of our assumption of $L S$ coupling. As we shall see, we observe $P_{2}$ to be zero within our experimental accuracy, meaning that any actual (small) breakdown of $L S$ coupling in the $\operatorname{Ar}$ II $\left(3 p^{4} 4 p\right)$ manifold (Table I) has negligible consequences for our analysis.
Continuing in the $L S$-coupling approximation, we may now generalize Eqs. (7) and (8) to decouple the individual $T(L)$ 's and $T(S)$ 's:

$$
\begin{aligned}
& \left\langle T(L)_{K Q}\right\rangle=\sum_{k q, k^{\prime} q^{\prime}} \beta_{K Q, k q, k^{\prime} q^{\prime}}^{L, l_{c}, l_{O}}\left\langle T\left(l_{c}\right)_{k q}\right\rangle\left\langle T\left(l_{o}\right)_{k^{\prime} q^{\prime}}\right\rangle, \\
& \left\langle T(S)_{K Q}\right\rangle=\sum_{k q, k^{\prime} q^{\prime}} \beta_{K Q, k q, k^{\prime} q^{\prime}}^{S, S_{c}, s_{O}}\left\langle T\left(s_{c}\right)_{k q}\right\rangle\left\langle T\left(s_{o}\right)_{k^{\prime} q^{\prime}}\right\rangle .
\end{aligned}
$$

This yields in turn the simultaneous equations for all relevant normalized multipole moments:

$$
\begin{aligned}
L(1)_{20} & =\frac{0.1000\left(l_{o}\right)_{20}+0.5917\left(l_{c}\right)_{20}+0.1196\left(l_{c}\right)_{20}\left(l_{o}\right)_{20}+0.9622\left(l_{c}\right)_{40}\left(l_{o}\right)_{20}}{1+0.5917\left(l_{c}\right)_{20}\left(l_{o}\right)_{20}}, \\
L(2)_{20} & =\frac{-0.5916\left(l_{o}\right)_{20}+0.5000\left(l_{c}\right)_{20}-0.5050\left(l_{c}\right)_{20}\left(l_{o}\right)_{20}-0.2710\left(l_{c}\right)_{40}\left(l_{o}\right)_{20}}{1-0.5916\left(l_{c}\right)_{20}\left(l_{o}\right)_{20}}, \\
L(3)_{20} & =\frac{0.4900\left(l_{o}\right)_{20}+0.8281\left(l_{c}\right)_{20}+0.1673\left(l_{c}\right)_{20}\left(l_{o}\right)_{20}+0.0374\left(l_{c}\right)_{40}\left(l_{o}\right)_{20}}{1+0.1691\left(l_{c}\right)_{20}\left(l_{o}\right)_{20}},
\end{aligned}
$$

and

$$
S_{11}=\left(s_{o}\right)_{11} .
$$

As will be discussed in Sec. IV, these simultaneous nonlinear equations must be solved to yield the elemental multipole moments.

We now consider briefly the effect of a breakdown in $L S$ coupling of the $3 p^{4}$ core. In the $3 p^{4} 4 p$ manifold the so-called $3 p^{4} 4 p$ "[ $\left.{ }^{1} D\right] 4 p^{2} P_{J}$ " states are actually a mixture composed of $\sim 85 \%{ }^{1} D$ core and $\sim 15 \%{ }^{3} P$ core (see Table I). Specifically, we must write

$$
\left.\mid \text { “ }\left({ }^{1} D\right) 4 p^{2} P_{3 / 2} ”\right\rangle=\alpha_{1}^{3 / 2}\left|\left({ }^{1} D\right) 4 p^{2} P_{3 / 2}\right\rangle+\alpha_{3}^{3 / 2}\left|\left({ }^{3} P\right) 4 p^{2} P_{3 / 2}\right\rangle
$$

and

$$
\begin{aligned}
\left.\mid \text { “ }\left({ }^{1} D\right) 4 p^{2} P_{1 / 2} "\right\rangle= & \alpha_{1}^{1 / 2}\left|\left({ }^{1} D\right) 4 p^{2} P_{1 / 2}\right\rangle \\
& +\alpha_{3}^{1 / 2}\left|\left({ }^{3} P\right) 4 p^{2} P_{1 / 2}\right\rangle,
\end{aligned}
$$

where the quotes indicate a non-Russell-Saunders state designated by approximate spectroscopic notation. [Note that, unlike the case of intermediate coupling in the first excited states of the neutral heavy noble gases, the expansion coefficients of Eqs. (19) are not symmetric because of other states that contribute to the expansion.] While Eqs. (16)-(18) for the $L=2$ and 3 states remain unchanged, Eqs. (13) and (14) must now be generalized to include the effect of intermediate coupling in the $L=1$ states [22]:

$$
\begin{aligned}
\left\langle T(L)_{K Q}\right\rangle_{J}= & \sum_{i j} \alpha_{i}^{J} \alpha_{j}^{J} \sum_{K Q, k q, k^{\prime} q^{\prime}} \beta_{K Q, k q, k^{\prime} q^{\prime}}^{L, l_{l}, l_{c,}, l_{c, j}}\left\langle T\left(l_{c, i}, l_{c, j}\right)_{k q}\right\rangle \\
& \times\left\langle T\left(l_{o}\right)_{k^{\prime} q^{\prime}}\right\rangle
\end{aligned}
$$

and

$$
\begin{aligned}
\left\langle T(S)_{K Q}\right\rangle_{J}= & \sum_{i j} \alpha_{i}^{J} \alpha_{j}^{J} \sum_{K Q, k q, k^{\prime} q^{\prime}} \beta_{K Q, k q, k^{\prime} q^{\prime}}^{S, s_{o}, s_{c, i}, s_{c}, j}\left(T\left(S_{c, i}, S_{c, j}\right)_{k q}\right\rangle \\
& \times\left\langle T\left(s_{o}\right)_{k^{\prime} q^{\prime}}\right\rangle,
\end{aligned}
$$

where

$$
\begin{aligned}
\beta_{K Q, k q, k^{\prime} q^{\prime}}^{X, x_{o}, x_{i,}, x_{c}, j}= & \sqrt{2 k+1} \sqrt{2 k^{\prime}+1}(2 X+1)\left(k^{\prime} q^{\prime}, k q \mid K Q\right) \\
& \times\left\{\begin{array}{ccc}
k & k^{\prime} & K \\
x_{c, j} & x_{o} & X \\
x_{c, i} & x_{o} & X
\end{array}\right\} .
\end{aligned}
$$

Equations (20)-(22) allow the possibility of complex "twocomponent" irreducible tensor multipole moments $\left\langle T\left(l_{c, i}, l_{c, j}\right)_{k q}\right\rangle$ and $\left\langle T\left(s_{c, i}, s_{c, j}\right)_{k q}\right\rangle$, corresponding to offdiagonal rectangular blocks of the $3 p^{4}$ core density matrix [22]. This has the unfortunate result that the number of normalized irreducible tensor multipole moments increases from four to ten: $l_{c}(1)_{20}, \operatorname{Re} l_{c}(1,2)_{20}, \quad \operatorname{Im} l_{c}(1,2)_{20}, s_{c}(1)_{11}$, $\operatorname{Re} s_{c}(1,0)_{11}$, and $\operatorname{Im} s_{c}(1,0)_{11}$ must now be included in the expansions of Eqs. (20) and (21). From the Stokes parameter measurements we would now determine independent values $L(1)_{20}, L(2)_{20}, L(3)_{20}, L(1)_{21}$, and $S(1 / 2)_{11}$ for $L=1$, and $S(1 / 2)_{11}$ for either $L=2$ or $L=3$, the latter two being depen- 
dent solely on $\left(s_{o}\right)_{11}$. These parameters depend in turn on the ten independent normalized subshell multipoles. Because $L$ and $S$ are each individually well-defined for a given $J$ state, we have sufficient information to determine $s_{o}(1 / 2)_{11}$, $s_{c}(1)_{11}, \operatorname{Re} s_{c}(1,0)_{11}$, and $\operatorname{Im} s_{c}(1,0)_{11}$, but lack by two parameters the information to determine all the $l_{c}$ and $l_{o}$ normalized multipoles. Thus a restriction of measurements to the " $\left({ }^{1} D\right) 4 p^{2} L_{J}$ " manifold alone allows full determination of the spin partitioning in the excitation, but precludes a complete experiment. Fortunately, mixing of the $3 p^{4}\left({ }^{3} P,{ }^{1} D\right) 4 p$ states with other configurations or states with a $3 p^{4}{ }^{1} S$ term is negligible [11]. Thus two additional Stokes parameter measurements of transitions from a doublet state with a predominantly ${ }^{3} P$ core would, in principle, allow us to determine all ten subshell multipoles. As we shall discuss in Sec. V, energy differences (and hence radial matrix elements) between the " $P$ " and " $D$ " core states cause this procedure to be problematic, but still potentially valid.

Given the relatively small level of ${ }^{3} P$ contribution to the core, and the fact that we measure no nonzero values of $P_{2}$ at our level of experimental precision (which would be a clear indication of the importance of the breakdown of $L S$ coupling), we proceed to use Eqs. (3)-(18).

\section{PREVIOUS RELATED WORK}

Although this is the first experimental work to succeed in extracting multipole moments of individual atomic subshells, a number of earlier investigations have provided similar or complementary information. Since the 1960s, optical excitation functions of various $\mathrm{Ar}$ II states produced by electron impact have been measured [7-10,23-27]. In the 1960s and 1970s, these experiments were driven in large part by the need for spectroscopic data basic to the design and construction of Ar ion lasers. Perhaps the most notable of the early experiments in the context of this work is that of Clout and Heddle [23], who made measurements of $P_{1}$ as a function of energy for a number of transitions. More recently, the Perth group has been particularly active, making integrated Stokes parameter measurements for simultaneous ionization and excitation of $\mathrm{Kr}$ [12] and $\mathrm{Zn}$ [28] by electron impact, as well as the first $(e, \gamma)$ coincidence measurements of (excitation +ionization) collisions with He targets [29]. The theory for such coincidence experiments was first developed by the Münster group [13]. The relative ease with which integrated measurements can be made compared with coincidence measurements is evident from the paucity of data from the latter. While the integration obscures some physics, the relative ease of data taking allows a much grater range of parameter space to be explored. Moreover, subshell information, unavailable in coincidence measurements, can be extracted from integrated experiments.

Another series of related investigations, carried out by Jaecks et al., has involved the (excitation+ionization) of $\mathrm{Ar}$ in charge transfer collisions with $\mathrm{He}^{+}$[30-33]. These experiments, in which scattered neutral He was detected, succeeded in measuring the electronic octupole moment components $L(3)_{31}$ and $L(3)_{33}$, as well as the rank-1 and rank-2 multipole moments of the $\operatorname{Ar}^{+*}\left[3 p^{4}\left({ }^{1} D\right) 4 p^{2} F\right]$ states. Without actually determining the individual subshell moments, they showed that the orientation of the orbital angular momentum $\left[L(3)_{11}\right]$ produced in the collision was consistent with complete orientation of the $p$ electron and no orientation of the ${ }^{1} D$ core over most impact parameters. It was this shell-specific analysis, discussed in Refs. [30,33], that inspired the present work. Jaecks et al. were also able to demonstrate the validity of the Rubin-Bederson hypothesis with regard to the fine structure components of the ${ }^{2} F$ state they considered. They did this by showing that $L(3)_{11}$ extracted separately from the $J=5 / 2$ and $J=7 / 2$ data were the same within experimental error.

Finally, the Perth group has made another series of investigations in which $\mathrm{H}$ and $\mathrm{He}$ are excited to $n=3$ states by electron impact. By making $(\gamma, \gamma)$ coincidence measurements and, in one case with $\mathrm{He},(2 \gamma, e)$ triple coincidence measurements, they were able to extract information about the excited state multipoles up to rank $4[34,35]$. In the latter experiment, essentially complete quantum mechanical information about the excited $3^{1} D$ state of $\mathrm{He}$ was obtained. These experiments are exceedingly difficult and, even in the best cases, yield values of the higher multipole moments that have large experimental uncertainty.

For the sake of completeness, we mention that a number of investigators have taken up the question of the validity of the Rubin-Bederson hypothesis. In situations where magnetic ("relativistic") forces are appreciable during the collision, as can be the case with high- $Z$ targets or when resonant states form an intermediary collision complex, one sees departures from this impulsive approximation [36,37].

\section{APPARATUS AND EXPERIMENTAL CONSIDERATIONS}

The apparatus used in these experiments has been described previously $[6,38,39]$ and is illustrated in Fig. 3. Briefly, photoemission from bulk GaAs is used to produce a beam of electrons with a transverse polarization of $\sim 20 \%$ and an energy width of $\sim 0.4 \mathrm{eV}$ FWHM. This beam, guided by both electrostatic and magnetic steering elements, traverses a differential pumping chamber and an isolation valve before entering a cylindrical target cell. This cell, nominally $5 \mathrm{~cm}$ in diameter, has a $1.0 \mathrm{~mm}$ diameter entrance aperture and a $2.0 \mathrm{~mm}$ exit aperture for the electron beam. At the top end of the cylinder is a lens whose focal point lies on the electron beam axis, and which serves as a vacuum wall. This beam is electrostatically shielded from the lens by a series of metal apertures. Electrons exiting the target cell are detected in a Faraday cup formed from several downstream electrostatic lens elements.

The Stokes parameters of the light emitted as a result of the electron-argon collisions [Eq. (1)] were measured using a polarimeter comprising the light gathering lens followed by a retarder, linear polarizer, interference filter, and a second planoconvex lens to focus the light onto a photomultiplier tube. Great care was taken in this experiment to characterize and understand the systematics of the polarimeter optical train. The retarder and polarizer were both placed in rotatable mounts so that the effects of local variations in their respective optical constants could be evaluated. The retardance and 


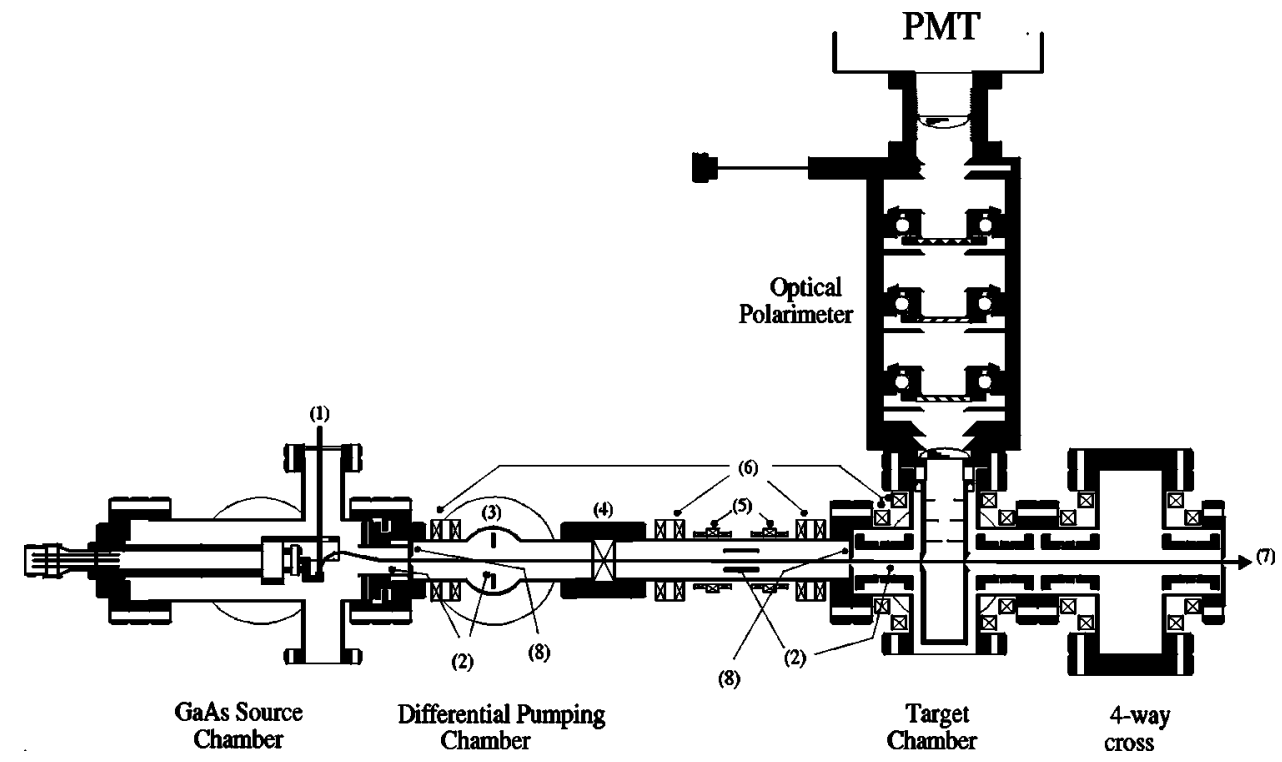

FIG. 3. Scale diagram of the apparatus used in this work. (1) $780 \mathrm{~nm}$ laser beam for photoemission of polarized electrons; (2) electrostatic steering and focusing elements; (3) differential pumping chamber; (4) isolation gate valve; (5) solenoidal spin rotators; (6) magnetic steering and field compensation coils; (7) transversely polarized electron beam; (8) beam defining differential pumping apertures. The optical polarimeter elements (moving downstream) are a 5-cm-diameter fused silica collection lens, rotatable retarder, rotatable linear polarizer, narrow-band interference filter, light gate valve, focusing lens, and photomultiplier tube (PMT).

polarizing efficiency of the various optical elements, averaged over their illuminated area, was determined using several complementary techniques which provided results in good agreement. (Uncertainties in our quoted data incorporate uncertainties in our knowledge of these quantities.)

Since we do not detect the scattered electrons, the interference filters used in the optical polarimeter serve to identify the excited ionic states produced in the collision. This technique has the advantages of very high energy resolution (as opposed to techniques based on electron energy spectroscopy) and relatively high count rates (as opposed to measurements differential in electron scattering angle), but suffers from the averaging inherent in an integral technique. An additional difficulty is that fluorescence arising from transitions in neutral argon with wavelengths close to those associated with the $3 p^{4}\left({ }^{1} D\right) 4 p$ Ar II manifold can contaminate the signal if it falls within the bandpass of the interference filter. This dictates the use of very narrow bandpass filters, but complete isolation can remain difficult because the optical excitation cross sections for Ar I are typically an order of magnitude higher than those for Ar II. We thus chose Ar II transitions that had (1) wavelengths longer than $400 \mathrm{~nm}$ to allow very narrow band (0.3-0.5 nm FWHM) interference filters to be manufactured; (2) the highest possible oscillator strengths; (3) the best possible wavelength isolation from Ar I transitions; (4) initial and final $J$ values that yielded reasonable Stokes parameter values for a given ionic alignment or orientation [see Eqs. (3)-(5)]; and (5) reasonable energy gaps between their threshold and the closest cascading threshold (see below).

In Table III, we show the initial and final states of the four transitions we chose based on these criteria, as well as their wavelengths, the respective interference filter center wavelengths and band widths, thresholds, the closest cascading

TABLE III. Transitions studied in this work.

\begin{tabular}{|c|c|c|c|c|c|c|}
\hline $\begin{array}{l}\text { Initial } \\
\text { state }\end{array}$ & $\begin{array}{l}\text { Final } \\
\text { state }\end{array}$ & $\begin{array}{l}\text { Wavelength } \\
\text { (nm) }\end{array}$ & $\begin{array}{c}\text { Filter central } \\
\text { wavelength } \\
(\mathrm{nm}) \\
(\mathrm{FWHM})\end{array}$ & $\begin{array}{c}\text { Threshold } \\
\text { energy } \\
(\mathrm{eV})\end{array}$ & $\begin{array}{c}\text { Closest } \\
\text { cascade } \\
\text { level }\end{array}$ & $\begin{array}{c}\text { Energy } \\
\text { gap } \\
(\mathrm{eV})\end{array}$ \\
\hline $\begin{array}{c}\left({ }^{1} D\right) 4 p \\
{ }^{2} F_{7 / 2}\end{array}$ & $\begin{array}{l}\left({ }^{1} D\right) 4 s \\
{ }^{2} D_{5 / 2}\end{array}$ & 460.96 & $460.8(4)$ & 36.90 & $\begin{array}{c}\left({ }^{1} D\right) 5 s \\
{ }^{2} D_{5 / 2}\end{array}$ & 3.14 \\
\hline $\begin{array}{l}\left({ }^{1} D\right) 4 p \\
{ }^{2} F_{5 / 2}\end{array}$ & $\begin{array}{l}\left({ }^{1} D\right) 4 s \\
{ }^{2} D_{5 / 2}\end{array}$ & 463.73 & $463.7(5)$ & 36.89 & $\begin{array}{c}\left({ }^{1} D\right) 5 s \\
{ }^{2} D_{5 / 2}\end{array}$ & 3.14 \\
\hline $\begin{array}{c}\left({ }^{1} D\right) 4 p \\
{ }^{2} D_{5 / 2}\end{array}$ & $\begin{array}{l}\left({ }^{1} P\right) 3 d \\
{ }^{2} D_{5 / 2}\end{array}$ & 448.18 & $448.3(3)$ & 37.26 & $\begin{array}{c}\left({ }^{1} D\right) 4 d \\
{ }^{2} D_{5 / 2}\end{array}$ & 3.26 \\
\hline $\begin{array}{c}\left({ }^{1} D\right) 4 p \\
{ }^{2} P_{3 / 2}\end{array}$ & $\begin{array}{c}\left(\begin{array}{l}1 \\
D\end{array}\right) 4 s \\
{ }^{2} D_{3 / 2}\end{array}$ & 423.73 & 423.7(4) & 37.11 & $\begin{array}{c}\left({ }^{1} D\right) 3 d \\
{ }^{2} S_{1 / 2}\end{array}$ & 1.46 \\
\hline
\end{tabular}




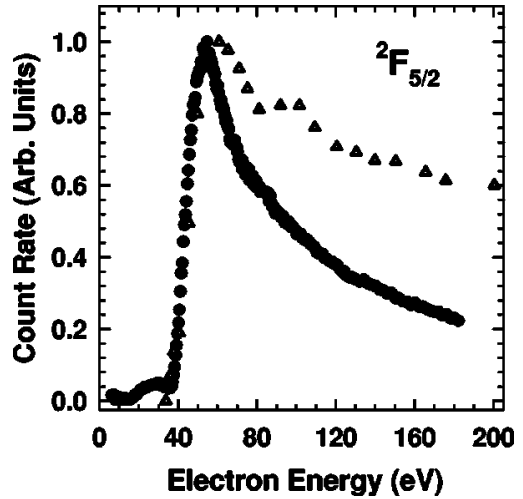

FIG. 4. Optical excitation functions for the ${ }^{2} F_{5 / 2}$ state. Solid circles: data of this work, taken with a target pressure of $3.0 \mathrm{mTorr}$, monitoring the $463.73 \mathrm{~nm}$ transition. The intensity is measured through a linear polarizer set at an angle of $54.8^{\circ}$ with respect to the beam axis [42] so that it does not depend on the alignment of the excited state. Open triangles: data of Ref. 9 for the $458.90 \mathrm{~nm}$ transition, taken with pressure between 0.7 and 5 mTorr (see text).

state and the energy gap between the excitation and closest cascade thresholds. As discussed above, measurement of $P_{1}$ and $P_{3}$ for only one transition from each $L$ manifold is required to completely determine the four nonzero subshell normalized multipole moments. We chose to study transitions from both of the $L=3$ fine-structure multiplets in order to provide redundant information about $L(3)_{20}$. If the values of this multipole are consistent for both transitions, we can infer that the $L$ and $S$ multipoles are formed in a time much shorter than the fine-structure relaxation time for this manifold.

Criterion (5) relating to cascading emphasizes an important limitation of our experiment. Because we did not measure the energies of the electrons emerging from the collision volume, we cannot discriminate against collisions in which the upper state in question is populated by cascade transitions (see Table III). Since we must combine data from the $P$, $D$, and at least one of the $F$ upper states to extract all of the multipoles in question we have, strictly speaking, complete cascade-free data sets available only in the energy range between $37.3 \mathrm{eV}$ and $38.6 \mathrm{eV}$. However, oscillator strengths of the $\mathrm{Ar}$ II transitions and conservative estimates of the probable populations of the upper cascading levels imply that there is no significant contamination of the ${ }^{2} P_{3 / 2}$ transition occurring for several $\mathrm{eV}$ above its first cascading threshold $[40,41]$. This allows a reasonable extension of the data set energy range to perhaps $41 \mathrm{eV}$.

The optimized selection of filters listed in Table III still did not isolate the $463.73 \mathrm{~nm}$ and $448.18 \mathrm{~nm}$ transitions completely from the Ar I contaminant lines at $462.8 \mathrm{~nm}$ and $448.1 \mathrm{~nm}$. These ArI lines produced a $30 \%$ and $60 \%$ background-to-signal ratio at $2 \mathrm{eV}$ above the threshold energy for production of the ${ }^{2} F_{5 / 2}$ and the ${ }^{2} D_{5 / 2}$ states, respectively. This contamination can be clearly seen between 15 and $35 \mathrm{eV}$ in the data of Fig. 4, the measured optical excitation function for the ${ }^{2} F_{5 / 2}$ state.

The potentially large sources of background, the small energy range above threshold over which we can make mea- surements that have negligible contributions from cascading, and the rather small polarization values we often observed made the subtraction of background a crucial step in our data analysis. Background can be divided into two types: that which depends on electron beam current and that which does not. The latter includes the dark noise of the PMT and stray light from a variety of sources. These two combined to produce a signal of 2-3 counts/s in our apparatus. Electroncurrent-dependent background can be caused by collisions between electrons and the metal walls of the target cell and electron optics that are close to it, as well as the abovementioned contamination from Ar I transitions. It is possible to determine the electron beam-wall collision background by turning the argon gas off and measuring the signal as a function of electron energy. Such experiments showed that this source of background was negligible.

Our raw data comprised a series of optical excitation functions obtained with different settings of the optical polarizer elements. For each one of these, we estimated the (beam + target)-related background by measuring its intensity at several energies, $E$, below threshold (covering an energy range equal to that range measured above the threshold) and then estimating the background intensity above threshold by extrapolation. For each setting, we fit the below-threshold intensity to a linear function of the form $I(E)=A+B E$. Data analysis proceeded by subtracting the beam-unrelated background from the total accumulated counts. The remaining signal was normalized to target pressure and the current transmitted through the target cell. Then, the extrapolated background at energies above the excitation threshold was subtracted with the appropriate propagation of errors. Finally, the different excitation functions were combined to determine the Stokes parameters as a function of energy for each transition.

Measurements of the optical excitation functions allowed us to calibrate the energy of the incident electrons. The energy of the electrons in the target cell did not correspond exactly to the potential difference between the crystal and the target cell. The contact potential variations between them are primarily responsible for this. To determine the absolute electron energy scale, we measured the voltage at which the (most intense) $461.0 \mathrm{~nm}$ transition exhibited a count rate that was statistically higher than the background rate. We found that the voltage supply that set the electron energy had a voltage shift of about $1.9 \mathrm{eV}$. There was no significant change in this value over time. All of the electron energies listed in this paper are corrected for this energy shift.

We investigated the effect of the target pressure on the polarization of the fluorescence radiation by measuring $P_{1}$ for all states in question at energies where the excitation cross section is large and the $P_{1}$ values are not significantly depolarized by cascading. Within statistical uncertainty, $P_{1}$ did not change for any of the transitions investigated over a pressure range between 0.5 and 3.0 mTorr. All of the measurements in this paper were made at 3.0 mTorr. In this context, however, we note that excitation functions that we measured exhibited significant pressure dependence. We attribute the difference between our data and that of Feltsan and Povich [9] in Fig. 4 to this effect. We will address these issues in a future paper. 


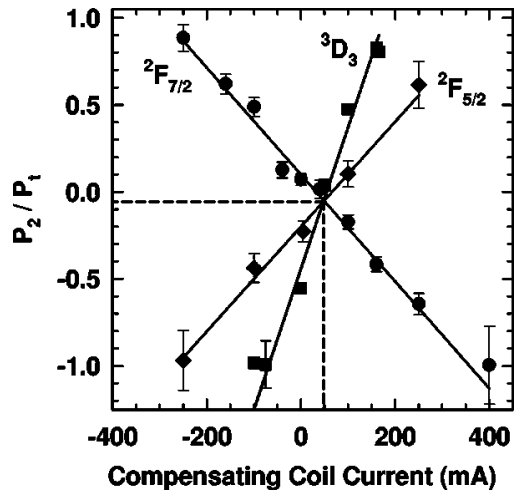

FIG. 5. Ratio of polarization fraction $P_{2}$ to the total linear polarization $P_{t}$ vs $I$, the current through the compensating Helmholtz coils. $P_{2}$ for zero $B$ field in the collision volume must equal zero. Data for ${ }^{2} F_{7 / 2}$ (circles) and ${ }^{2} F_{5 / 2}$ (diamonds) transitions in Ar II, as well as the ${ }^{3} D_{3}{ }^{-} P_{2} 811.5 \mathrm{~nm}$ transition (squares) in Ar I (see text).

Another serious potential source of systematic error was spurious magnetic fields in the target chamber. The turbo pump beneath the target cell is the main source of these fields. Such fields can alter the collision geometry by deflecting the incident electron beam and/or causing Hanle depolarization of the emitted fluorescence $[38,43,44]$. To study possible effects due to Hanle depolarization, we measured the linear polarization of the fluorescence for three excited states of $\mathrm{Ar}$ (one for $\mathrm{Ar}$ I and two for $\mathrm{Ar}$ II) as a function of the magnetic field $B$ parallel to the fluorescence direction. If one assumes that the field-free value of $P_{2}$ is zero, one can show that $[43]$

$$
\frac{P_{2 m}}{P_{1}}=\frac{2 \gamma \omega_{0}}{\sqrt{4 \gamma^{2} \omega_{0}^{2}+\gamma^{4}}},
$$

where $P_{2 m}$ is the measured value of $P_{2}$ at a given magnetic field, $P_{1}$ is the field-free value, $\gamma$ is the excited-state decay constant, and $\omega_{0}$ is the Larmor precession frequency of the excited state:

$$
\omega_{L}=g_{J} \frac{e B}{2 m},
$$

where $g_{J}$ is the gyromagnetic ratio of the upper state of the transition and $e$ and $m$ are the electron charge and mass, respectively. Thus the Hanle-induced value of $P_{2 m}$ is a sensitive measure of the magnetic field in the collision volume.

In order to minimize magnetic fields in the collision region, two solenoidal coils in an approximate Helmholtz configuration were placed outside the vacuum region of the target cell, below and above the beam line (see Fig. 3). Their symmetry axes were coincident with the axis of the optical polarimeter. Because it was difficult to measure the magnetic field in the interaction region directly, we zeroed the field using the measured ratio of $P_{2 m}$ to $P_{1}$, using unpolarized incident electrons to guarantee that $P_{2}=0$. This was done for the ${ }^{2} F_{7 / 2}$ and ${ }^{2} F_{5 / 2}$ transitions in $\mathrm{Ar}$ II, as well as the $811.5 \mathrm{~nm}, 3 p^{5} 4 p^{3} D_{3} \rightarrow 2 p^{5} 4 s^{3} P_{2}$ transition in Ar I (Fig. 5). While the slopes of $P_{2 m}$ vs coil current are different due to the various gyromagnetic ratios of the various upper states (as well as the quantum numbers of the lower states), all three data sets have a common zero, corresponding to a coil current of $\sim 50 \mathrm{~mA}$, or a $65 \mu \mathrm{T}$ compensating field. This "triple intersection" method proved to be a highly sensitive way to eliminate the vertical $B$ field in the collision volume.

Measuring the polarization of the incident electrons, $P_{e}$, is necessary for normalization of the $P_{2}$ and $P_{3}$ data. We determined $P_{e}$ immediately before and after taking the data reported here by measuring the integrated Stokes parameters $P_{1}$ and $P_{3}$ for the resonance fluorescence at $811.5 \mathrm{~nm}$ of the ${ }^{3} D_{3}$ state of $\mathrm{Ar}$ I [45]. About eight months elapsed during this time. All the data reported here were taken using the same GaAs crystal with several heat cleanings and activations being made over the course of the work. We found $P_{e}$ to be unchanged over this time interval, varying from $20.2 \pm 0.3 \%$ to $20.0 \pm 0.5 \%$. We thus used an average value of $20.1 \pm 0.3 \%$ for normalization purposes. This value is significantly lower than earlier measurements made by our group with both a different apparatus [20] and the same [38] apparatus used here. The values of $P_{e}$ determined in those references were $27 \%$ and $28 \%$, respectively. The present polarization value may be due to anomalous conditions for this crystal. During heat cleaning, there was a white film on its surface that we could not remove by increasing its temperature. This film might have played a role in depolarizing the photoemitted electrons.

We used the $2 s 2 p^{2}{ }^{2} D$ negative-ion resonance $(58.85 \mathrm{eV})$ in $\mathrm{He}$ to measure the energy distribution of the electron beam. This resonance can decay to the $1 s 3 d^{3} D$ state with a natural linewidth $\Gamma=0.025(10) \mathrm{eV}$ [38]. Since this width is very small in comparison with the energy spread of the GaAs

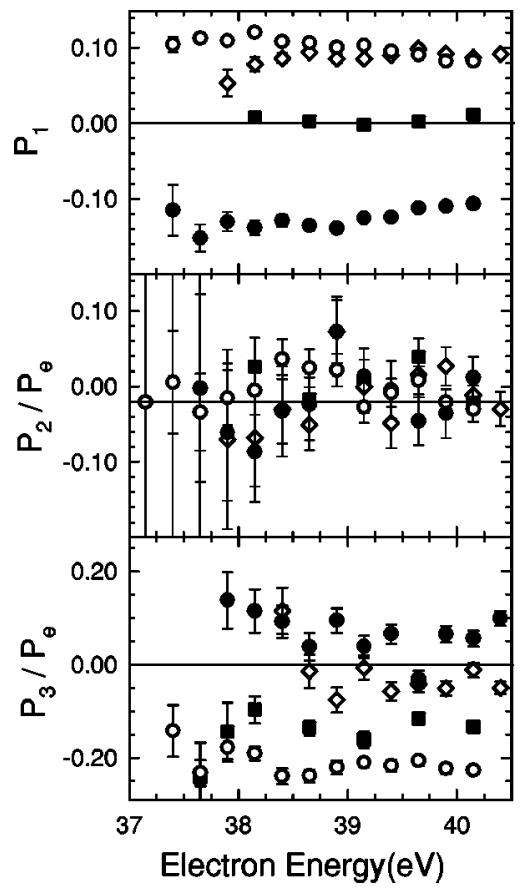

FIG. 6. Stokes parameters for the four transitions investigated, indicated with $1 \sigma$ error bars. Spin-dependent Stokes parameters are normalized to incident electron polarization. Data are for the upper states ${ }^{2} F_{7 / 2}$ (open circles), ${ }^{2} F_{5 / 2}$ (solid circles), ${ }^{2} D_{5 / 2}$ (diamonds), and ${ }^{2} P_{3 / 2}$ (squares). 


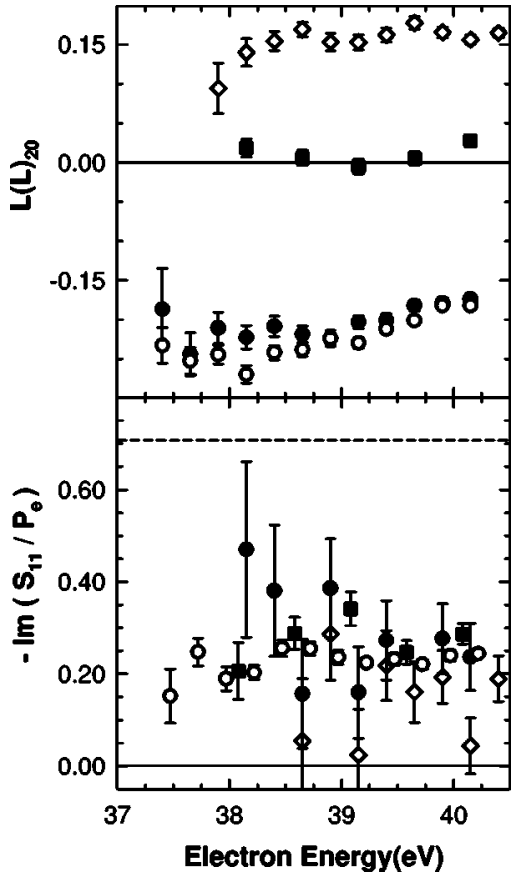

FIG. 7. Derived $L$ state normalized electric quadrupole moments and $S$ magnetic dipole moments (see text). Symbol legend is the same as in Fig. 6. Dashed line corresponds to pure exchange excitation of the $4 p$ subshell (see text).

electron source, the width of the resonance feature gives a good estimate of the energy resolution of our experiment. Using this method we found our electron beam to have a typical energy width of $0.40(5) \mathrm{eV}$.

\section{DATA AND ANALYSIS}

The Stokes parameter data for all four transitions are shown in Fig. 6. We note that, within the $1 \sigma$ uncertainty of the data, the normalized values of $P_{2}$ are nil for all transitions. Using the data of Fig. 6 and Eqs. (11) and (12), we can extract the values of $L(L)_{20}$ and $-\operatorname{Im}\left\{S_{11} / P_{e}\right\}$. These are plotted in Fig. 7.

The fact that the $L(3)_{20}$ values are essentially independent of the $J$ multiplet from which they are derived validates the Rubin-Bederson picture of the $L$ and $S$ multipoles "setting up" before significant intra-atomic spin-orbit coupling has occurred. Even stronger evidence for this time scale is provided by the fact that $S_{11}$ is the same for all the states we studied within the statistical uncertainty of the data. This implies that the magnetic dipole of the residual ion subshells are determined during or soon after the collision, before the total $L$ and $S$ multipoles have time to form.

Because of the nonlinear nature of Eqs. (15)-(17), some care must be taken in determining the values and uncertainties of the $\left(l_{c}\right)_{k q}$ and $\left(l_{o}\right)_{k q}$ from the $L(L)_{k q}$. To address this issue we combined terrain search and Monte Carlo algorithms. Determination of the uncertainties is particularly difficult. The standard propagation of errors technique has a significant drawback in that it can give misleading results if

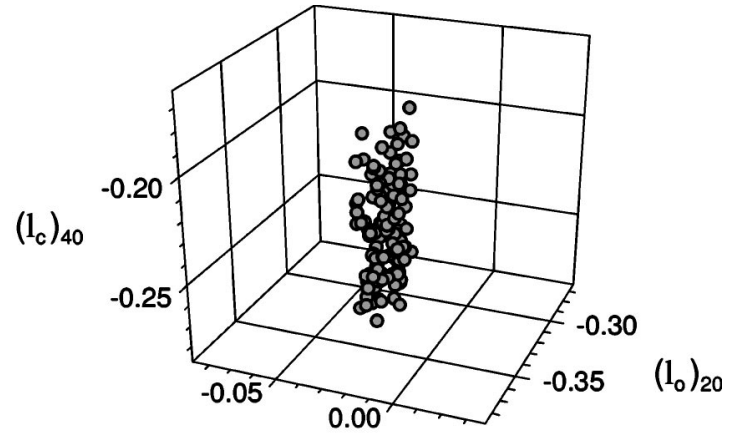

$\left(1_{c}\right)_{20}$

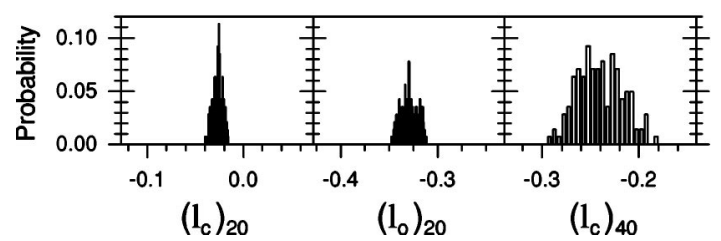

FIG. 8. Distribution of the solutions of Eqs. (15)-(17) about their global minimum for an incident electron energy of $40.2 \mathrm{eV}$ (see text). The histograms are the projections of the data on the $l$-space axes.

the function that must be evaluated is nonlinear near the solution point. The Monte Carlo (MC) method, on the other hand, does not require any knowledge or prior assumptions about the function that must be inverted [46]. Our method relies on generating a set of artificial data points that mimics the statistics of each measurement of the $L(L)_{k q}$ 's (i.e., the mean value and its uncertainty), and inverting the equation that relates this value to the $l_{k q}$ 's for each one of these artificial data points. The distribution of the solutions $\left[\left(l_{c}\right)_{20}\right.$, $\left(l_{c}\right)_{40}$, and $\left.\left(l_{o}\right)_{20}\right]$ forms a "solution cloud" in $l$ space. The standard deviation of this cloud about its mean in the solution space corresponds to the uncertainties in these derived multipoles. For this method to work, the artificial set must be statistically indistinguishable from the parent distribution from which the actual measured value was drawn. The uncertainties that we used for the $L_{k q}$ 's were derived from the accumulated photon counts.

To invert Eqs. (15)-(17), we used a terrain search algorithm. As the name implies, this algorithm searches the do-

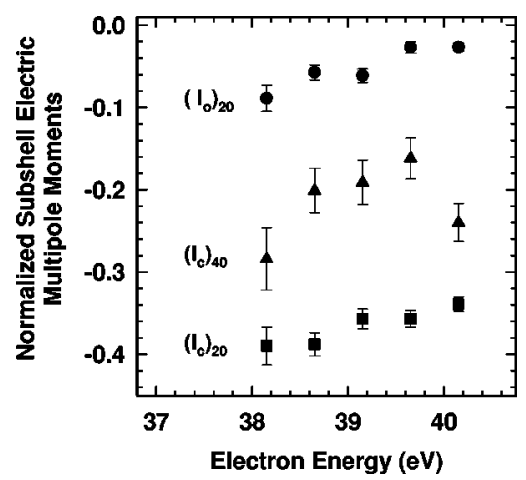

FIG. 9. Normalized subshell electric multipole moments for the core and outer electron. 


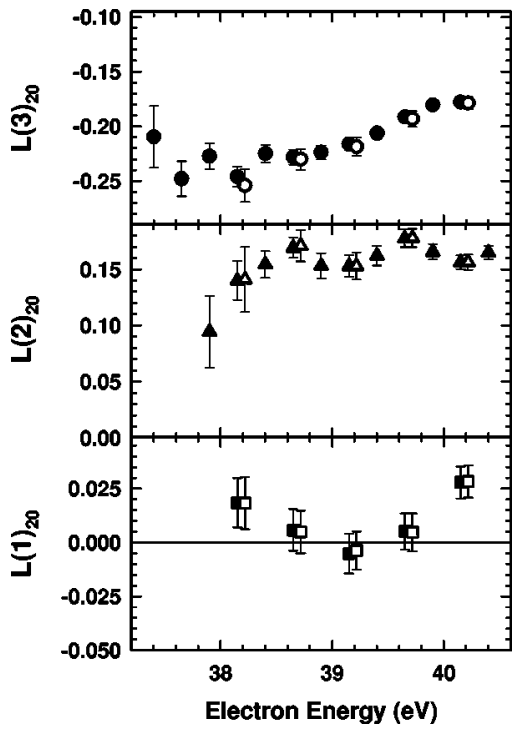

FIG. 10. Normalized electric quadrupole moments of the ${ }^{2} P$ (squares), ${ }^{2} D$ (triangles), and ${ }^{2} F$ (circles) states. Solid symbols represent the measured data; open symbols are the results obtained by recoupling the individual subshell multipole moments. The five comparable sets of reconstructed and measured data have been offset slightly from each other on the energy scale.

main of a scalar function until it finds a local minimum. For our scalar function, $d$, we used the Euclidean distance between the vector of the measured relative orbital multipoles, $\vec{u}=\left\{L(3)_{20}, L(2)_{20}, L(1)_{20}\right\}$, and the vector of the relative multipole values estimated from the individual electron multipoles, $\quad \vec{v}=\left\{F_{1}\left[\left(l_{c}\right)_{20},\left(l_{c}\right)_{40},\left(l_{o}\right)_{20}\right] ; F_{2}\left[\left(l_{c}\right)_{20},\left(l_{c}\right)_{40},\left(l_{o}\right)_{20}\right]\right.$; $\left.F_{3}\left[\left(l_{c}\right)_{20},\left(l_{c}\right)_{40},\left(l_{o}\right)_{20}\right]\right\}$ obtained from Eqs. (15)-(17) and weighted by the uncertainty in the measured values. To make sure we found the global minimum of $d$, we started from a number random points throughout the entire allowed space of $\vec{u}$, which is bounded by angular momentum constraints.

Figure 8 is an example of the typical "solution cloud" that we obtained for each energy we investigated. The mean value and width of these distributions correspond to the derived $l$ 's and their uncertainties. All of these solutions are unimodal, compact, and reasonably well described by Gaussian functions at all energies, implying that they are robust and well defined. The results of this analysis are shown in Fig. 9. The individual values of $\left(l_{c}\right)_{20},\left(l_{c}\right)_{40}$, and $\left(l_{o}\right)_{20}$ can now be plugged back into Eqs. (13) and (15)-(17) to generate the recoupled "derived" values of $L(1)_{20}, L(2)_{20}, L(2)_{40}$,

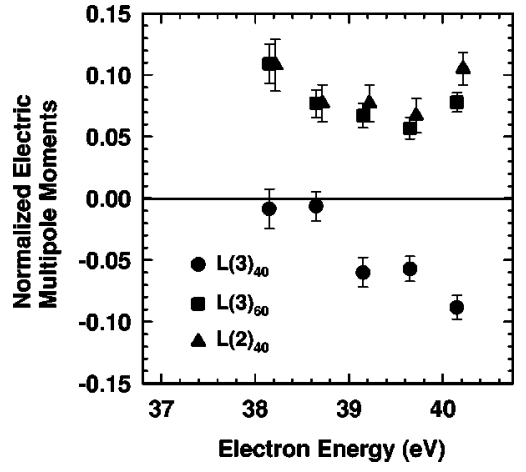

FIG. 11. Normalized electric hexadecapole and hexacontatetrapole moments of the ionic system.

$L(3)_{20}, L(3)_{40}$, and $L(3)_{60}$. Thus by making a series of singlephoton measurements, we can extract information about rank-4 (hexadecapole) and rank-6 (hexacontatetrapole) moments of an atomic system within the framework of a given angular momentum coupling scheme. To make these measurements directly would require two- and three-photon coincidence measurements, respectively. These recoupled moments are shown in Figs. 10 and 11. Note that the measured values of the $L(Y)_{20}$, obtained directly from Eq. (11), can be compared with the "derived" values (Fig. 10). The two data sets are consistent with each other, which gives us further confidence that our inversion method for extraction of the individual subshell multipole moments is correct.

\section{DISCUSSION AND CONCLUSIONS}

The fact that $L(3)_{20}$ is independent of the multiplet component from which it is obtained provides experimental evidence for the validity of the RB hypothesis on a time scale longer than $\sim 3 \times 10^{-14} \mathrm{~s}$. In other words, we have demonstrated experimentally that multipoles of $L$ and $S$ are not correlated for the ${ }^{1} D$ core configuration we are studying. The collision-time arguments made at the end of Sec. II allow one to reasonably argue that the multipoles of $l_{c}$ and $l_{o}$ ought to be uncorrelated as well, i.e., that Eqs. (15)-(17) are valid. Unfortunately, we have no direct experimental way to check this assertion because no redundant coupling equations exist for $l_{c}$ and $l_{o}$. If the multipoles of $l_{c}$ and $l_{o}$ are correlated, we must write their products in Eq. (13) as $\left\langle T\left(l_{c}\right)_{k q} \otimes T\left(l_{o}\right)_{k^{\prime} q^{\prime}}\right\rangle$ instead of $\left\langle T\left(l_{c}\right)_{k q}\right\rangle\left\langle T\left(l_{o}\right)_{k^{\prime} q^{\prime}}\right\rangle$. This leads to equations of the type

$$
L(X)_{20}=\frac{a\left\langle\left(l_{c}\right)_{00} \otimes\left(l_{o}\right)_{20}\right\rangle+b\left\langle\left(l_{c}\right)_{20} \otimes\left(l_{o}\right)_{00}\right\rangle+c\left\langle\left(l_{c}\right)_{20} \otimes\left(l_{o}\right)_{20}\right\rangle+d\left\langle\left(l_{c}\right)_{40} \otimes\left(l_{o}\right)_{20}\right\rangle}{1+e\left\langle\left(l_{c}\right)_{20} \otimes\left(l_{o}\right)_{20}\right\rangle,}
$$

replacing Eqs. (15)-(17), where

$$
\left\langle\left(l_{c}\right)_{k q} \otimes\left(l_{o}\right)_{k^{\prime} q^{\prime}}\right\rangle \equiv \frac{\left\langle T\left(l_{c}\right)_{k q} \otimes T\left(l_{o}\right)_{k^{\prime} q^{\prime}}\right\rangle}{\left\langle T\left(l_{c}\right)_{00} \otimes T\left(l_{o}\right)_{00}\right\rangle} .
$$




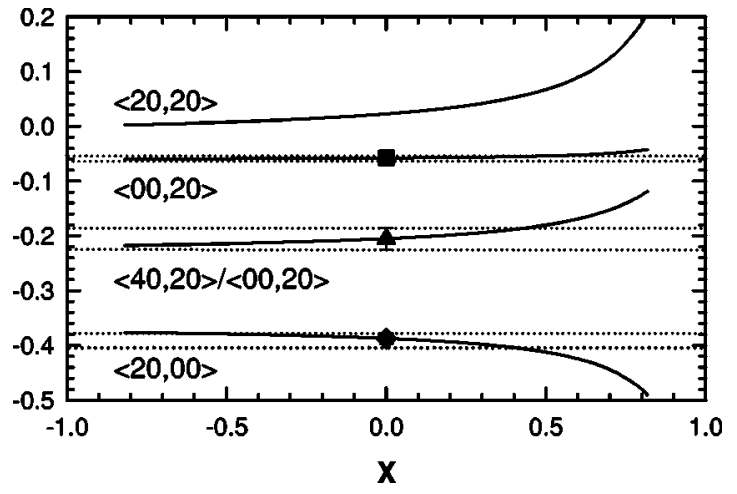

FIG. 12. Values of correlated multipoles as a function of the correlation parameter $x$. When $x=0$, the lower three correlated quantities on the graph are measurable (see text). The size of the error bars corresponding to the core and outer electric multipole measurements excludes $x$ values greater than about 0.5 .

We are now confronted with four independent variables instead of three, but still have only three independent measurements: $P_{1}$ for either multiplet from each $L$ component of the manifold.

How do possible correlations between subshell multipole moments affect our results? We are insensitive to correlations solely between $\left(l_{c}\right)_{40}$ and $\left(l_{o}\right)_{20}$. In this case, there are still only three independent variables, with $\left\langle\left(l_{c}\right)_{40} \otimes\left(l_{o}\right)_{20}\right\rangle$ simply replacing $\left(l_{c}\right)_{40}$. We can explore how sensitive our measurements are to possible correlations between $\left(l_{c}\right)_{20}$ and $\left(l_{c}\right)_{20}$ by constructing a "correlation parameter"

$$
x=\frac{\langle 20,20\rangle-\langle 20,00\rangle\langle 00,20\rangle}{\langle 20,20\rangle+\langle 20,00\rangle\langle 00,20\rangle},
$$

where

$$
\left\langle k q, k^{\prime} q^{\prime}\right\rangle \equiv\left\langle\left(l_{c}\right)_{k q} \otimes\left(l_{o}\right)_{k^{\prime} q^{\prime}}\right\rangle
$$

This parameter equals zero for no correlation and ranges from -1 for pure anticorrelation to +1 for pure correlation. When $x=0$, the correlated multipole combinations $\langle 20,00\rangle$, $\langle 00,20\rangle$, and $\langle 40,20\rangle /\langle 00,20\rangle$ reduce to $\left(l_{c}\right)_{20},\left(l_{o}\right)_{20}$, and $\left(l_{c}\right)_{40}$, respectively. Figure 12 shows the values of the correlated multipoles over the domain of $x$. Note that the denominator of $x$ in Eq. (27) is positive definite for our data set.

Given the statistical accuracy of our experiment, it is obvious that we are insensitive to possible correlations between $\left(l_{c}\right)_{20}$ and $\left(l_{o}\right)_{20}$ at the level necessary to cause $x<0.5$. In other words, our results can exclude correlation only over the range $0.5<x<1$. With this caveat, we will assume for the rest of our discussion that the core and $4 p$ multipoles are uncorrelated.

Putting correlation issues aside, one must also critically evaluate the effects of cascading and excitation dynamics on the values of the extracted subshell multipoles. From Table III, we see that cascading from higher-lying states can, in principle, contaminate the data above $38.6 \mathrm{eV}$. Given the lower excitation cross sections for these upper states, especially close to their respective thresholds, gives us confi-
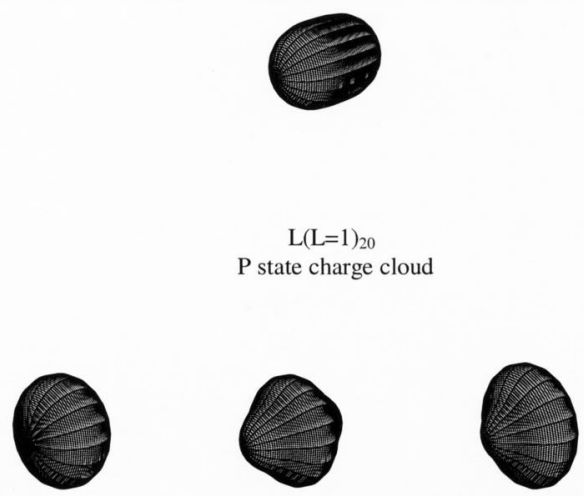

$\mathrm{L}(\mathrm{L}=2)_{20}$

$\mathrm{L}(\mathrm{L}=2)_{40}$

D state charge cloud
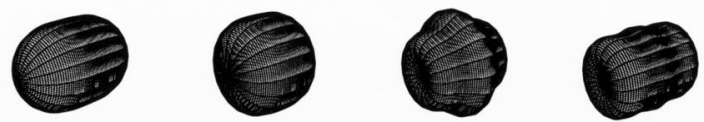

$\mathrm{L}(\mathrm{L}=3)_{20}$

$\mathrm{L}(\mathrm{L}=3)_{40}$

$\mathrm{L}(\mathrm{L}=3)_{60}$

F state charge cloud

$\mathrm{E}=38.15 \mathrm{eV}$

(a)

$$
\begin{array}{r}
\mathrm{L}(\mathrm{L}=1)_{20} \\
\end{array}
$$

P state charge cloud
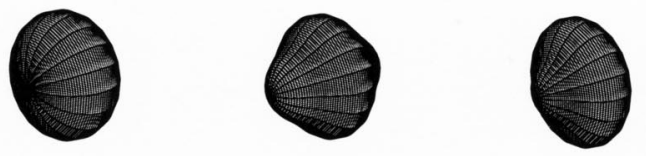

$\mathrm{L}(\mathrm{L}=2)_{20}$

$\mathrm{L}(\mathrm{L}=2)_{40}$

D state charge cloud
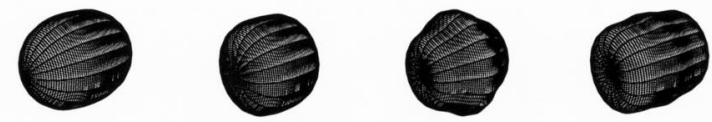

$\mathrm{L}(\mathrm{L}=3)_{20}$

$\mathrm{L}(\mathrm{L}=3)_{40}$

$\mathrm{L}(\mathrm{L}=3)_{60}$

F state Charge cloud

$E=40.15 \mathrm{eV}$

(b)

FIG. 13. Contributions of the $L$ multipoles to the charge cloud density of the $P, D$, and $F$ states at 38.15 and $40.15 \mathrm{eV}$. 
dence that contamination should not be significant below $41 \mathrm{eV}$. We have extracted values up to $40.2 \mathrm{eV}$.

A potentially more serious problem is that the different excited states studied in this work have different threshold energies themselves. Thus one might expect that the dynamical conditions leading to the production of the core and $4 p$ electron would produce different multipole values for each of these states. Let us consider the extreme case of the data taken at $38.2 \mathrm{eV}$. At this incident energy, the asymptotic speed of the ionized and projectile electrons (assuming, for the sake of discussion, equal energy sharing) varies by $18 \%$ between the ${ }^{2} F$ states and the ${ }^{2} D$ state. This concern is ameliorated by our observation that the multipole moments of the core and outer electron depend only weakly on energy. Moreover, the $18 \%$ difference is for the asymptotic speeds; the speeds near the residual ion as the electrons leave the collision volume will be closer to each other. As the incident electron energy increases, these differences become even smaller. All of these concerns are endemic to the integrated Stokes parameter measurement technique. While our data is probably influenced only minimally at our level of statistical accuracy by these effects, they must be kept in mind. The much cleaner double or triple coincidence technique [34,35] suffers from none of these problems, but has the difficulty that the data are much less precise given their typical count rates $[34,35]$.

The data on the normalized magnetic dipole moment of the $4 p$ electron allow us to estimate the importance of exchange collisions for populating this shell. The horizontaldashed line in the lower half of Fig. 7 corresponds to a value of $-\operatorname{Im}\left\{S_{11} / P_{e}\right\}=1 / \sqrt{2}$. This would be the experimental result if the excited outer electron was produced exclusively through exchange with the incident polarized beam. In pure exchange scattering (no spin flips) of the polarized electron with spin up $(\uparrow)$ polarization (along the $y$ axis), for example, the following possibilities for the spin configuration of the $3 p^{4}$ core electrons, the $4 p$ excited electron, and the ejected electrons are possible:
(We are studying states that have a ${ }^{1} D$ core, so the core electrons have to be paired to give zero total spin angular momentum.) Pure exchange population of the $4 p$ electron dictates that only the first two configurations listed above can occur. Thus the expectation value of the Cartesian spin component of the excited state is

$$
\left\langle\left(s_{o}\right)_{y}\right\rangle=\frac{1}{2} \hbar P_{e} .
$$

Now for electrons [43]

$$
\left\langle T_{1 Q}\left(s_{o}\right)\right\rangle=\sqrt{2}\left\langle\left(s_{o}\right)_{Q}\right\rangle \sigma
$$

and

$$
\sigma=\sqrt{2}\left\langle T_{00}\left(s_{o}\right)\right\rangle,
$$

where $\sigma$ is the total cross section and $Q=0, \pm 1$. Thus we have

$$
\left(s_{o}\right)_{1 Q}=2\left\langle\left(s_{o}\right)_{Q}\right\rangle .
$$

For a particle with spin $s$, the Cartesian components of the polarization vector $P_{i}$ with respect to the expectation value of its Cartesian spin components $\left\langle s_{i}\right\rangle$ are given by

$$
\left(P_{e}\right)_{i}=\frac{\left\langle s_{i}\right\rangle}{s} .
$$

Transforming Cartesian components into spherical ones, we write

$$
s_{1 Q}=\left\{\begin{array}{l}
-\frac{1}{\sqrt{2}}\left( \pm P_{x}+i P_{y}\right), \quad Q= \pm 1, \\
P_{z}, \quad Q=0 .
\end{array}\right.
$$

Since in our experiment we have $P_{x}=P_{z}=0, P_{y}=P_{e}$,

$$
\frac{\left(s_{o}\right)_{11}}{P_{e}} \equiv-\frac{\left(s_{o}\right)_{1-1}}{P_{e}}=-\frac{i}{\sqrt{2}} .
$$

Thus the maximum possible value of $-\operatorname{Im}\left(s_{o}\right)_{11} / P_{e}$, assuming pure exchange with the outer electron, is $1 / \sqrt{2}=0.71$. The measured value of the $-\operatorname{Im}\left(s_{o}\right)_{11} / P_{e}$ is considerably smaller than this value, and is $\approx 0.25 \pm 0.10$. This means that there are more dominant collision channels, including core exchange and/or direct excitation of the $4 p$ outer electron.

We note in passing that the electric multipoles of the various $L$ terms can be written in terms of the excitation cross sections for their $m_{L}$ magnetic sublevels, $\sigma_{m_{L}}$, where $\sigma_{m_{L}}$ $=\sigma_{-m_{L}}$ by symmetry:

$$
\begin{gathered}
L(1)_{20}=-\sqrt{\frac{2}{3}} \sigma_{0}+\sqrt{\frac{2}{3}} \sigma_{ \pm 1}, \\
L(2)_{20}=-\sqrt{\frac{2}{7}} \sigma_{0}-\sqrt{\frac{2}{7}} \sigma_{ \pm 1}+\sqrt{\frac{8}{7}} \sigma_{ \pm 2},
\end{gathered}
$$




$$
\begin{gathered}
L(2)_{40}=\sqrt{\frac{18}{35}} \sigma_{0}-\sqrt{\frac{32}{35}} \sigma_{ \pm 1}+\sqrt{\frac{2}{35}} \sigma_{ \pm 2}, \\
L(3)_{20}=-\sqrt{\frac{4}{21}} \sigma_{0}-\sqrt{\frac{3}{7}} \sigma_{ \pm 1}+\sqrt{\frac{25}{21}} \sigma_{ \pm 3}, \\
L(3)_{40}=\sqrt{\frac{18}{77}} \sigma_{0}+\sqrt{\frac{2}{77}} \sigma_{ \pm 1}-\sqrt{\frac{14}{11}} \sigma_{ \pm 2}+\sqrt{\frac{18}{77}} \sigma_{ \pm 3},
\end{gathered}
$$

and

$$
L(3)_{60}=-\frac{10}{\sqrt{231}} \sigma_{0}+\sqrt{\frac{75}{77}} \sigma_{ \pm 1}-\sqrt{\frac{12}{77}} \sigma_{ \pm 2}+\frac{1}{\sqrt{231}} \sigma_{ \pm 3} .
$$

Knowing the state multipoles allows us to determine $W_{L}$, the electron angular charge distribution of the excited states [43]:

$$
W_{L}(\theta, \varphi)=\frac{1}{\sqrt{4 \pi}} \sum_{k q}(-1)^{L}(2 L+1)\left(\begin{array}{ccc}
L & L & k \\
0 & 0 & 0
\end{array}\right) L(L)_{k q} Y_{k q}(\theta, \varphi),
$$

where the third term in the sum represents $3 \mathrm{~J}$ coefficients and the $Y_{k q}(\theta, \varphi)$ are the spherical harmonics. Using this equation, we can determine the contribution of each multi- pole (by assuming zero for the others) as well as the total linear combination of them. Due to the weak energy dependence of the $4 p$ outer electron and core electric multipoles, the angular charge cloud distributions do not change significantly over the energy range that we investigated. For this reason, we show these distributions only at 38.15 and $40.15 \mathrm{eV}$ (Fig. 13). It is apparent from the figure that the higher-order moments of rank 4 and 6 play a significant role in determining the shape of the excited state charge cloud for the $\mathrm{Ar}^{+*}$ residual ion. Thus any complete analysis of these collision systems must include a description of the subshell multipoles from which these higher order moments are constructed.

In the work reported here, the role of the polarized electrons is limited to verifying the Rubin-Bederson hypothesis for the $S$ multipoles, and to providing some insight about the role of exchange in the excitation of the $4 p$ electron of the residual ion. The next logical step for this work is to investigate $\mathrm{Ar}$ II states with a ${ }^{3} P$ core in conjunction with the $4 p$ outer electron. One could then hope to investigate spin partitioning between the subshells as well as the distribution of orbital angular momentum.

\section{ACKNOWLEDGMENT}

This work was supported by a Grant from the National Science Foundation (NSF PHY-0099363).
[1] E. W. McDaniel, Atomic Collisions: Electron and Photon Projectiles (Wiley, New York, 1989).

[2] N. Andersen and K. Bartschat, Polarization, Alignment, and Orientation in Atomic Collisions (Springer, New York, 2001).

[3] T. N. Rescigno, M. Baertschy, W. A. Isaacs, and C. W. McCurdy, Science 286, 2474 (1999).

[4] D. V. Fursa, and I. Bray, Phys. Rev. A 52, 1279 (1995).

[5] V. Zeman, K. Bartschat, T. J. Gay, and K. W. Trantham, Phys. Rev. Lett. 79, 1825 (1997).

[6] H. M. Al-Khateeb, B. G. Birdsey, and T. J. Gay, Phys. Rev. Lett. 85, 4040 (2000).

[7] W. B. Bridge, Appl. Phys. Lett. 4, 128 (1964).

[8] W. R. Bennett, Jr., G. N. Mercer, P. J. Kindlmann, B. Wexler and H. Hyman, Phys. Rev. Lett. 17, 987 (1970).

[9] P. V. Feltsan and M. M. Povch, Opt. Spectrosc. 28, 119 (1970).

[10] K.-H. Tan and J. W. McConkey, Phys. Rev. A 10, 1212 (1974).

[11] H. Statz, F. A. Horrigan, S. H. Koozekanani, C. L. Tang, and G. F. Koster, J. Appl. Phys. 36, 2278 (1965).

[12] P. A. Hayes, D. H. Yu and J. F. Williams, J. Phys. B 31, L193 (1998).

[13] R. Schwienhorst, A. Raeker, K. Bartschat, and K. Blum, J. Phys. B 29, 2305 (1996).

[14] K. Rubin, B. Bederson, M. Goldstein, and R. E. Collins, Phys. Rev. 182, 201 (1969).

[15] I. C. Percival and M. J. Seaton, Philos. Trans. R. Soc. London, Ser. A 251, 113 (1958).

[16] G. Csanak, S. Trajmar, J. C. Nickel, G. F. Hanne, J. W. Mc-
Conkey, T. J. Gay, and M. A. Khakoo, Comments At. Mol. Phys. 30, 165 (1994).

[17] C. Cohen-Tannoudji, B. Diu, and F. Laloe, Quantum Mechanics (Wiley, New York, 1977), p. 250.

[18] B. F. J. Luyken, Physica (Amsterdam) 60, 432 (1972).

[19] K. Bartschat and K. Blum, Z. Phys. A 304, 85 (1982).

[20] J. E. Furst, W. M. K. P. Wijayaratna, D. H. Madison, and T. J. Gay, Phys. Rev. A 47, 3775 (1993).

[21] K. Bartschat, K. Blum, G. F. Hanne, and J. Kessler, J. Phys. B 14, 3761 (1981).

[22] D. H. Yu, P. A. Hayes, J. F. Williams, V. Zeman, and K. Bartschat, J. Phys. B 33, 1881 (2000).

[23] P. N. Clout and D. W. O. Heddle, J. Phys. B 4, 483 (1971).

[24] I. D. Latimer and R. M. St. John, Phys. Rev. A 1, 1612 (1970).

[25] I. P. Bogdanova and S. V. Yurgenson, Opt. Spectrosc. 62, 21 (1987).

[26] J. A. Sánchez, J. A. Aguilera, B. Martínez, and J. Campos, Phys. Rev. A 41, 1392 (1990).

[27] S. Tsurubuchi, J. Phys. Soc. Jpn. 66, 3070 (1996).

[28] D. H. Yu, L. Pravica, J. F. Williams, N. Warrington, and P. A. Hayes, J. Phys. B 34, 3899 (2001).

[29] P. A. Hayes and J. F. Williams, Phys. Rev. Lett. 77, 3098 (1996).

[30] B. W. Moudry, O. Yenen, and D. H. Jaecks, Phys. Rev. Lett. 71, 991 (1993).

[31] B. W. Moudry, O. Yenen, and D. H. Jaecks, Z. Phys. D: At., Mol. Clusters 30, 199 (1993).

[32] O. Yenen, B. W. Moudry, and D. H. Jaecks, Can. J. Phys. 74, 
955 (1996).

[33] B. W. Moudry, O. Yenen, D. H. Jaecks, and J. H. Macek, Phys. Rev. A 54, 4119 (1996).

[34] J. F. Williams, M. Kumar, and A. T. Stelbovics, Phys. Rev. Lett. 70, 1240 (1993).

[35] A. G. Mikosza, in The Physics of Electronic and Atomic Collisions, XXI International Conference, edited by Y. Itikawa, K. Okuno, H. Tanaka, A. Yagishita, and M. Matsuzawa, AIP Conf. Proc. No. 500 (American Institute of Physics, Melville, NY, 2000), p. 297.

[36] C. W. McLucas, W. R. MacGillivray, and M. C. Standage, Phys. Rev. Lett. 48, 88 (1982).

[37] K. Bartschat, U. Thumm, and D. W. Norcross, J. Phys. B 25, L641 (1992).

[38] B. G. Birdsey, H. M. Al-Khateeb, M. E. Johnston, T. C. Bowen, T. J. Gay, V. Zeman, and K. Bartschat, Phys. Rev. A 60, 1046 (1999).

[39] H. M. Al-Khateeb, B. G. Birdsey, T. C. Bowen, A. S. Green,
M. E. Johnston, and T. J. Gay, Rev. Sci. Instrum. 70, 3882 (1999).

[40] A. Hibbert and J. E. Hansen, J. Phys. B 27, 3325 (1994).

[41] W. L. Wiese, M. W. Smith, and B. M. Mile, Atomic Transitions Probabilities, Natl. Stand. Ref. Data Ser., Natl. Bur. Stand. (U.S.), Circ. No. 22 (U.S. GPO, Washington, D.C., 1969), Vol. II.

[42] P. N. Clout and D. W. O. Heddle, J. Opt. Soc. Am. 59, 715 (1969).

[43] K. Blum, Density Matrix Theory and Applications, 2nd ed. (Plenum, New York, 1996).

[44] A. Corney, Atomic and Laser Spectroscopy (Oxford, New York, 1977).

[45] T. J. Gay, J. E. Furst, K. W. Trantham, and W. M. K. P. Wijayaratna, Phys. Rev. A 53, 1623 (1996).

[46] J. M. Chambers, Computational Methods for Data Analysis (Wiley, New York, 1977). 